\title{
127. SEGUNDA LOCALIDAD DE NARCISSUS CAVANILLESII A. BARRA \& G. LÓPEZ (AMARYLLIDACEAE) PARA PORTUGAL
}

\author{
Antònia ROSSELLÓ-GRAELL, Isabel MARQUES y David DRAPER
}

\begin{abstract}
Second locality of Narcissus cavanillesii A. Barra \& G. López (Amaryllidaceae) for Portugal.
\end{abstract}
Palabras llave. Amaryllidaceae, Narcissus, corología, conservación, embalse del Alqueva, Portugal.

Key words. Amaryllidaceae, Narcissus, chorology, conservation, Alqueva dam, Portugal.

Debido a la construcción en Portugal del embalse del Alqueva en el río Guadiana (Alto Alentejo) dio inicio en 1999 un proyecto de monitorización (Ballester - Hernández et al., 2000) con el objetivo de evaluar el impacto de esta obra en las siguientes especies: Armeria neglecta Girard., Biarum galiani Talavera, Cheilanthes acrosticha (Balbis) Tod., Cistus psilosepalus Sweet., Cynara tournefortii Boiss. \& Reuter, Dianthus lusitanus Brot., Eryngium galioides Lam., Flueggea tinctoria (L.) G. L. Webster, Isoetes delilei Rothm., Linaria hirta (L.) Moench, L. ricardoi Cout., Luzula forsteri (Sm.) DC. in Lam. \& DC., Marsilea batardae Launert, Narcissus cavanillesii A. Barra \& G. López, N. serotinus L., Nerium oleander L., Picris willkommii (Schultz Bip.) Nyman, Salix salvifolia Brot. subsp. australis Franco y Sanguisorba hybrida (L.) Nordborg. Las prospecciones de campo realizadas en el marco de este proyecto se centraron en el tramo del río Guadiana comprendido entre la frontera con España y la población de Moura. Dichas prospecciones se realizaron, para las especies otoñales, entre los meses de septiembre y diciembre de 1999 y permitieron encontrar la segunda localidad de Narcissus cavanillesii A. Barra \& G. López para Portugal en el municipio de Alandroal (Alto Alentejo).

Esta especie ibero-mauritánica está referenciada para las provincias españolas de Cádiz, Córdoba, Huelva, Sevilla (Valdés et al., 1987), Málaga (MA 22495) y Badajoz (Devesa, 1995; Rivas Goday \& Ladero Álvarez, 1973). En el continente africano su presencia está citada para Argelia y Marruecos (Maire, 1959; Valdés et al., 1987). Barra (2002) descarta la cita para la provincia de Cáceres al considerarla un error de identificación.

El hecho de que la más reciente flora portuguesa (Franco \& Rocha Afonso, 1994) no considere esta especie puede posiblemente deberse a un olvido de los autores ya que Malato-Beliz (1977) refirió la existencia de $N$. cavanillesii en Ajuda (Elvas, Alto Alentejo), 29SPC5993, siendo esta la primera localidad conocida en Portugal para esta especie.

En el presente trabajo se aporta la segunda localidad de $N$. cavanillesii para Portugal ampliando el ámbito corológico conocido de este taxon en la Península Ibérica.

Narcissus cavanillesii A. Barra \& G. López in Anales Jard. Bot. Madrid 41(1): 202 (1984). PORTUGAL, ALTO ALENTEJO: Alandroal, Montes Juntos, Herdade da Defesa, Monte Novo de Cima. 29SPC4664, 127 m, solo compactado, afloramentos rochosos, 15-X1999, David Draper, Alq 262. (LISU 173700).

Esta nueva población se sitúa a unos 30

Proyecto promovido por EDIA, S. A. y cofinanciado por EDIA, S. A. y por FEDER. 
$\mathrm{km}$ al sur de la anteriormente conocida y también en el lecho del río Guadiana. La población se diferencia de las poblaciones españolas, visitadas por los autores en otoño de 2000, por su grado de agregación ya que los individuos se encuentran agrupados en un conjunto de diez núcleos de tamaño variable (de $0.3-10 \mathrm{~m}^{2}$ ) a lo largo de $500 \mathrm{~m}$ del río Guadiana con escasos individuos aislados. En las poblaciones visitadas los individuos se encuentran dispersos en núcleos de gran superficie pero con una baja densidad de individuos. El substrato está formado por afloramientos de rocas de esquisto y una matriz arcillosa alóctona de sedimentación aportada por el río. $N$. cavanillesii se establece tanto en las grietas de las rocas, sobre el suelo arcilloso o en situaciones mixtas (arcilla-roca). El tamaño poblacional estimado es de ca. 1500 individuos reproductores.

A la vez cabe destacar que en la otra población portuguesa situada en Elvas, con ca. 12000 individuos en total, éstos se reparten en dos núcleos principales de $150 \mathrm{~m}^{2}$ y $6 \mathrm{~m}^{2}$ correspondiendo el núcleo mayor (85\% de esta población) a la parte superior de las ruinas del puente de Ajuda.

Las dos poblaciones portuguesas de $N$. cavanillesii se encuentran dentro de la zona de influencia del embalse del Alqueva quedando la de Montes Juntos totalmente sumergida. El bajo número de efectivos poblacionales de esta especie en Portugal, la elevada concentración de individuos en escasos metros de superficie, el impacto directo del embalse del Alqueva conjuntamente con el hecho de $N$. cavanillesii formar parte de los Anexos II y IV de la Directiva Hábitats 92/43/CEE y la zona que ocupa en Portugal estar incluida en la "Lista Nacional de Sitios de la Red Natura 2000: Sitio Guadiana-Juromenha" llevaron al diseño del proyecto "Proposta de salvaguarda de Narcissus cavanillesii A. Barra \& G. López como medida de minimização da construção da barragem do Alqueva" promovido por la empresa que construye y gestiona el embalse, EDIA, S. A. Este proyecto, iniciado en 2001, tiene como principal objetivo evitar la extinción de $N$. cavanillesii en Portugal y garantizar la viabilidad de sus poblaciones. Esta iniciativa engloba una serie de actuaciones dentro de la disciplina de la biología de la conservación que permitan asegurar los objetivos propuestos entre las cuales se incluye el rescate (traslocación) de la población de Montes Juntos para evitar la perdida de una de las dos poblaciones de $N$. cavanillesii en Portugal.

\section{BIBLIOGRAFÍA}

BALLESTER-HERNÁNDEZ, S., A. ROSSELLÓGRAELL, D. DRAPER \& A.I.D. CORREIA 2000- Monitorização de plantas prioritárias na área da albufeira do Alqueva. Portugalia Acta Biol. 19: 201-218.

BARRA, A. -2002- Notas sobre Narcissus L. (Amaryllidaceae), II. Anales Jard. Bot. Madrid 59(2): 351 .

DEVESA, J. -1995- Vegetación y Flora de Extremadura: 638. Universitas Editorial, Badajoz.

FRANCO, J. \& M. AFONSO -1994- Nova Flora de Portugal (Continente e Açores). AlismataceaeIridaceae. Vol.III. Fasc. I. Lisboa.

MAIRE, R. -1959-Flore de l'Afrique du Nord. Vol. VI: 76-77. Lechevalier. Paris.

MALATO-BELIZ, J. -1977- Braxireon humile (Cav.) Rafin-Nouvelle Amaryllidacée pour le Portugal. Candollea 32: 249-254.

RIVAS GODAY, S. y M. LADERO ÁLVAREZ 1973- Nuevas aportaciones a la flora pacense. Anales Real Acad. Farm. 3: 283.

VALDÉS, B., S. TALAVERA y E. FERNÁNDEZGALIANO - 1987- Flora Vascular de Andalucía Occidental Vol. III: 472. Ketres Editora S. A., Barcelona.

Aceptado para su publicación en octubre de 2003

Dirección de los autores. Jardim Botânico. Museu Nacional de História Natural. Rua da Escola Politécnica, 58. 1250-102, Lisboa. Portugal. 